For reprint orders, please contact: reprints@futuremedicine.com

\title{
Ending AIDS and challenges for Asia
}

Pirapon Ohata ${ }^{* 1}$, Ruksina Chumchure', Kesdao Nanthapisal', Chavalun Ruengpanyathip', Prapon Koita', Thitisan Phuanglek', Anchalee Avihingsanon ${ }^{1,2}$, Thanyawee Puthanakit ${ }^{1,3}$, Nittaya Phanuphak ${ }^{1,4}$, Stephen J Kerr ${ }^{1,5}$, Torsak Bunupuradah', Wasana Prasitsuebsai', Nadia Kancheva Landolt', Tavitiya Sudjaritruk', Vorapot Sapsirisavat', Tanya Do', Chatsuda Auchieng', Kiat Ruxrungtham ${ }^{1,2}$ \& Praphan Phanuphak ${ }^{1,2,4}$

The 17th Bangkok International Symposium on HIV Medicine, Queen Sirikit National Convention Centre, Bangkok, Thailand, 14-16 January 2015

HIV Netherlands Australia Thailand Research Collaboration (HIV-NAT)'s commitment to provide educational training every January to the region returned this year after the cancellation of 2014's symposium due to political unrest. More than 500 participants from five continents attended the 3-whole-day symposium; 60 also attended Data Safety and Monitoring Board (DSMB) preconference workshop sponsored by Harvard University's Multiregional Clinical Trial Center and 50 attended the Qualitative Research preconference workshop held by our sister organization SEARCH. A wide number of topics were discussed and a few are listed: prevention and cure, combination of antiretroviral therapy, elderly, coinfections, policy implementation, sexual health and stigma. This article briefly summarizes some of the plenary sessions.

Professor Joep Lange \& Jacqueline van Tongeren Memorial Lecture: treatment as prevention: approaches to maximize impact

Julio Montaner (British Columbia Centre for Excellence in HIV/AIDS and Centre for Excellence in HIV/AIDS at Providence Health Care, Canada) provided useful data, experiences and outcomes/ results [1] in the implementation of the treatment as prevention (Tas $\mathrm{P}$ ) [2,3] in various cities in Canada [4]. An example of the effectiveness of this strategy in reducing new infections was shown using data obtained from people who inject drugs. The 90-90-90 UNAIDS vision to eliminate HIV infection is achievable if implemented globally without contributing to more drug resistances [5] and was found to be cost effective in the long run.

'HIV-NAT, Thai Red Cross - AIDS Research Centre (TRCARC), Bangkok, Thailand

2Division of Allergy \& Immunology, Faculty of Medicine, Chulalongkorn University, Bangkok, Thailand

${ }^{3}$ Department of Pediatrics, Faculty of Medicine, Chulalongkorn University, Bangkok, Thailand

${ }^{4}$ SEARCH, Thai Red Cross - AIDS Research Centre (TRCARC), Bangkok, Thailand

5The Kirby Institute for Infection \& Immunity in Society, University of New South Wales, Sydney, NSW 2052, Australia

*Author for correspondence: Tel.: +662 652 3040; Fax: +662 252 579; juneohata4@gmail.com
Future

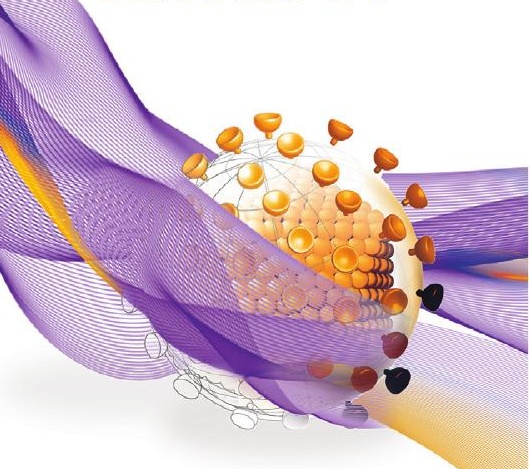

\section{KEYWORDS}

- Asia • cART • combination antiretroviral therapy

- elderly • HIV • PMTCT

- pre-exposure prophylaxis

- PrEP • prevention

of mother-to-child

transmission $\bullet$ public

policies $\bullet$ treatment

as prevention (TasP) 


\section{HIV \& aging: what clinicians should} not miss?

Peter Reiss (Division of Infectious Diseases, Department of Global Health Amsterdam Institute for Global Health and Development Academic Medical Center, University of Amsterdam, The Netherlands) presented evidence that there are increasing numbers of people living with HIV (PLWH) developing age-associated noncommunicable comorbidities such as osteopenia/osteoporosis (i.e., lumbar spine, femoral neck and total hip) [6], hypertension [7] and frailty (i.e., unintentional weight loss, weakness, exhaustion, slowness and low physical activity) [8]. The increase correlates with time spent at low CD4 counts [9]. He presented a comprehensive list of risk factors associated with comorbidities (i.e., toxicity from combination antiretroviral therapy [cART], lifestyles and genetics of the host, persistent immune dysregulation and inflammation in treated HIV disease) [10]. For example, smoking has a detrimental effect on subclinical atherosclerosis in older HIV-infected patients [11], causes loss of more life years [12] and contributes to mortality [13]. He gave examples of 'hallmarks of aging' [14] and acknowledged that toxicities from new antiretroviral drugs are less of a problem than those seen with older agents. For example, tenofovir alafenamide has less effect on bone mineral density than tenofovir disoproxil fumarate (TDF) [15].

\section{Adult HIV treatment guidelines: what's new?}

Ploenchan Chetchotsakd (Division of Infectious Diseases and Tropical Diseases, Faculty of Medicine, Khon Kaen University, Thailand) provided a comprehensive review of the recent changes of treatment guidelines comparing Department of Health and Human Services (DHHS), International AIDS Society USA, Europe, British HIV Association and Thai National Treatment Guidelines on their recommendations regarding optimal timing of antiretroviral therapy initiation, preferred cART regimens, Efavirenz in pregnancy and when to start antiretrovirals (ARVs) in HIV/tuberculosis coinfected populations. She presented data from relevant trials supporting the modified sections of the treatment guidelines such as STARTMRK, ACTG 5257, SINGLE, FLAMINGO, CIPRA HT 001 and HPTN 052.

\section{Treatment failure}

David Cooper (The Kirby Institute for Infection and Immunity in Society, Australia) comprehensively examined ways to manage and approach treatment failure by first discussing the WHO 2013 recommended regimens, as well as other relevant guidelines. He anticipates that in the future, first-line cART may use low-dose Efavirenz which has been shown to be safe and efficacious [16]. Other ideal characteristics would be low potential for interaction with rifampicin, available as a fixed-dose combination, and includes an integrase inhibitor. Studies have shown that virological failure is not a great method to measure failure in the low-income setting [17] and had a small benefit to monitor failure based on the intention-totreat [18] data. According to the WHO 2013, Zidovudine $(A Z T)$ can replace failed TDF or FTC whereas TDF can be used in place of failed AZT or Lamivudine (3TC). WHO's recommendation for the second-line regimens is not evidence based on clinical trials, but on the experiences of the experts. Lopinavir/ ritonavir (LPV/r) monotherapy is not recommended [19] and boosted protease inhibitors (PIs) also have resistance problems [20]. In order to keep matters simple without the need for genotype testing, he recommended the use of two new classes, or integrase inhibitor with boosted Lopinavir (LPV) for second-line regimens.

\section{The latest in prevention mother-to- child transmission \& pediatric treatment guidelines}

Thanyawee Puthanakit (Division of Infectious Diseases, Department of Pediatrics, Faculty of Medicine, Chulalongkorn University, Thailand) started the session by supporting the UNAIDS 2014 vision that in the future, all children will be born HIV free. She showed the evolution of the WHO's Prevention MotherTo-Child Transmission (PMTCT) guidelines spanning from 2001, 2004, 2006, 2010 and 2013 as well as from British HIV Association 2012, Thailand 2014 and DDHS 2014. She briefly mentioned that the new WHO PMTCT guidelines for 2015 should address challenging issues regarding MTCT prevention/HIV elimination; PMTCT service delivery/multiple entry points; ongoing risk of HIV in pregnant postpartum women; and early infant diagnosis/linkage to pediatric treatment. She then gave an extensive review of pediatric HIV treatment guidelines. She described critical issues related to providing treatment to infants, children and 
adolescents as well as provided a list of pediatric formulated ARVs currently available and also described the role of integrase inhibitors for both children and adolescents.

\section{Coordinating public policies for ending AIDS: the experience from Thailand}

Lessons learned from Thailand's HIV experiences and techniques used to develop/implement public policies, were explained by Nakorn Premsri (Department of Disease Control, Ministry of Public Health, Thailand). Time to initiate cART in Thailand was used as an example how an idea can go from paper to becoming part of the national guidelines. He provided step-by-step instructions how this was derived and implemented. The vision for AIDS-free generation from the International AIDS Conference 2012 inspired the Thai policy makers to form five committed working groups in various fields knowledgeable about HIV (i.e., modeling group/cost-effectiveness for TasP, scale-up, task-shifting for HIV test and counseling, option $\mathrm{B}+$ [long-term ARVs for mothers] and Pre-Exposure Prophylaxis $[\mathrm{PrEP}])$. This resulted in a series of consultations with TasP model, cost-benefit analysis/return of investment and the data from HPTN 052 study. Technical and economic evidence were presented to the policy makers, and convinced them that treatment for all would be the country's strategic plan to end AIDS. A strategic framework was developed to address the issues of task-shifting for HIV testing and counseling, strengthening the current cascade for treatment and care, as well as funding.

\section{Effect of stigma on HIV treatment uptake, retention \& care}

This issue has never been discussed at the symposium and was presented by Stephen J Kerr (HIV-NAT, Thailand; The Kirby Institute, Australia). It highlighted that stigma is very real in our society, and stems from poor and/or inaccurate knowledge about transmission, and fear of contagion. Stigma leads to people being treated differently, and discriminated against. Various kinds of stigma exist, including selfstigma (self-blame/guilt), perceived stigma and enacted stigma. These together result in stigma concern which has detrimental effects on the mental health of PLWH and is linked with poor adherence [21]. Stigma also presents people from marginalized groups from presenting for
HIV testing. Positive relationships with healthcare teams and social capital help to negate the effects of stigma. In Thailand, novel use of social media (e.g., Adam's Love website [22]) has helped to encourage men who have sex with men to present for HIV testing. Stigma reduction is an imperative component of 'getting to zero'.

\section{The great debate: starting antiretroviral therapy regardless of CD4 count is feasible in Southeast Asia}

Thailand has moved to treat all HIV-infected patients regardless of their CD4 count. Since many countries in the region look to and rely on the Thai National Treatment Guidelines, it was important to gauge whether other countries in the region would be prepared to adopt the same policy. The debate was facilitated by Annette Sohn (TREAT Asia, amfAR, Thailand). The audience members voted prior to the debate, with $57.1 \%$ agreeing that it would be feasible to treat all in Southeast Asia, 28.6\% disagreeing and $14.3 \%$ undecided. The 'yes' team, comprising Praphan Phanuphak (Thai Red Cross, AIDS Research Centre) and Julio Montaner stated that the benefits of early antiretroviral therapy are invaluable (e.g., recovery of immune function and ability to maximize the chances for a normal life span). They reminded the audience about their moral obligation to provide the highest quality of treatment available, which has been supported by multiple research studies. In addition, offering testing and then immediate early treatment had the potential to bring more people 'out of the woodwork' and into HIV testing. It was estimated by their team that the change from the current WHO-recommended treatment criteria to treating all patients with HIV accounted for only a $10 \%$ increase in patients, and thus in overall program costs. A healthier population would increase income per household, and reduce HIV transmission, death, longterm costs and drug resistance. Representing the team against the 'feasibility' were Sean Emery (The Kirby Institute, Australia) and Somnuek Sungkanuparph (Division of Infectious Diseases Department of Medicine, Faculty of Medicine, Ramathibodi Hospital, Mahidol University, Thailand). They argued that 'having a choice' is not the same as 'no choice to start ART'. All available data informing this issue are from observational cohort studies and scale-up is impossible if current ART coverage is less than $50 \%$ in Indonesia, Myanmar and Malaysia. 
Furthermore, treating people at all CD 4 counts may also lead to increases in drug resistance. They were able to convince a large proportion of the audience to change their decisions to 'unfeasible,' winning the vote by impressively increasing the percentage twofold to $56.6 \%$, whereas the 'yes' vote declined to $30.3 \%$ and undecided maintaining their neutrality at $13.1 \%$.

The 18th Bangkok International Symposium on HIV Medicine will be held on 13-15 January 2016.

\footnotetext{
Acknowledgements

The authors would like to thank all of the chairpersons, speakers and moderators for their continued support in providing the most up-to-date information to the symposium. The authors are extremely grateful to all of their sponsors, collaborators and partners to make this event possible. The authors would also like to thank the participants who attended the event and provided feedback so that we can work on improving the program. Last but not
}

the least, this symposium would not have been possible without the dedicated hard work and strong commitment from the symposium team for developing the scientific program to organizing, coordinating and running the show.

\section{Financial \& competing interests disclosure}

KRuxrungtham has served as a consultant for Merck and Tibotec; honoraria from Bristol-Meyers Squibb, Merck, Roche, Jensen-Cilag, GlaxoSmithKline and GPO; and educational grant for the Senior Researcher Scholar from the Thai Research Fund; and research grants from the National Research University Project of CHE and the Ratchadaphiseksomphot Endowment Fund (HR1161A). The rest of the authors have no other relevant affiliations or financial involvement with any organization or entity with a financial interest in or financial conflict with the subject matter or materials discussed in the manuscript apart from those disclosed.

No writing assistance was utilized in the production of this manuscript.

\section{References}

1 Samji H, Taha T, Moore D et al. Predictors of unstructured antiretroviral treatment interruption and resumption among HIV-positive individuals in Canada. HIV Med. 16(2), 76-87 (2015).

2 Montaner JS, Lima VD, Barrios R et al. Association of highly active antiretroviral therapy coverage, population viral load, and yearly new HIV diagnoses in British Columbia, Canada: a population-based study. Lancet 376(9740), 532-539 (2010).

3 Montaner JS, Lima VD, Harrigan PR et al. Expansion of HAART coverage is associated with sustained decreases in HIV/AIDS morbidity, mortality and HIV transmission: the "HIV Treatment as Prevention" experience in a Canadian setting. PLoS ONE 9(2), e87872 (2014).

4 Hogg RS, Nosyk B, Harrigan PR et al. Rates of new infections in British Columbia continue to decline at a faster rate than in other Canadian regions. HIV Med. 14(9), 581-582 (2013).

5 Werb D, Mills EJ, Montaner JS, Wood E. Risk of resistance to highly active antiretroviral therapy among HIV-positive injecting drug users: a meta-analysis. Lancet Infect. Dis. 10 (7), 464-469 (2010).

6 Schouten J, Wit FW, Stolte IG et al. Cross-sectional comparison of the prevalence of age-associated comorbidities and their risk factors between HIV-infected and uninfected individuals: the AGEhIV cohort study. Clin Infect Dis. 59(12), 1787-1797 (2014).

7 Kooij KW, Wit FW, Bisschop PH et al. Low bone mineral density in patients with well-suppressed HIV infection: association with body weight, smoking, and prior advanced HIV disease. J. Infect. Dis. 211(4), 539-548 (2014).

8 Van Zoest RA, Wit FW, Kooij KW et al. Higher prevalence of hypertension in HIV-infected patients partially explained by increased waist-hip ratio rather than BMI, other traditional risk factors or markers of systemic inflammation. Presented at: 16th International Workshop on Co-morbidities and Adverse Drug Reactions in HIV Workshop. Pennsylvania, SA, USA, 6-8 October 2014.

9 Kooij KW, Wit FWNM, Schouten J, van der Valk M, Stolte I, Reiss P. HIV infection is independently associated with frailty in middle-aged HIV-infected individuals compared with uninfected controls. Presented at: 8th Netherlands Conference on HIV Pathogenesis, Epidemiology, Prevention and Treatment. Amsterdam, The Netherlands, 18 November 2014.

10 Deeks SG, Phillips AN. HIV infection, antiretroviral treatment, ageing, and non-AIDS related morbidity. BMJ 338, a3172 (2009).

11 Fitch KV, Looby SE, Rope A et al. Effects of aging and smoking on carotid intima-media thickness in HIV-infection. AIDS 27(1), 49-57 (2013).

12 Helleberg M, May MT, Ingle SM et al. Smoking and life expectancy among
HIV-infected individuals on antiretroviral therapy in Europe and North America. AIDS 29(2), 221-229 (2015)

13 Helleberg M, Afzal S, Kronborg G et al. Mortality attributable to smoking among HIV-1-infected individuals: a nationwide, population-based cohort study. Clin. Infect. Dis. 56(5), 727-734 (2013).

14 Lopez-Otin C, Blasco MA, Partridge L, Serrano M, Kroemer G. The hallmarks of aging. Cell 153(6), 1194-1217 (2013).

15 Sax PE, DeJesus E, Mills A et al. Coformulated elvitegravir, cobicistat, emtricitabine, and tenofovir versus co-formulated efavirenz, emtricitabine, and tenofovir for initial treatment of HIV-1 infection: a randomised, double-blind, phase 3 trial, analysis of results after 48 weeks. Lancet 379(9835), 2439-2448 (2012).

16 Group, ES, Puls, R, Amin, J et al. Efficacy of $400 \mathrm{mg}$ efavirenz versus standard $600 \mathrm{mg}$ dose in HIV-infected, antiretroviral-naive adults (ENCORE1): a randomised, double-blind, placebo-controlled, non-inferiority trial. Lancet 383(9927), 1474-1482 (2014).

17 Rawizza HE, Chaplin B, Meloni ST et al. Immunologic criteria are poor predictors of virologic outcome: implications for HIV treatment monitoring in resource-limited settings. Clin Infect Dis. 53(12), 1283-1290 (2011).

18 Mermin J, Ekwaru JP, Were W et al. Utility of routine viral load, CD 4 cell count, and 
clinical monitoring among adults with HIV receiving antiretroviral therapy in Uganda: randomised trial. BMJ. 343, d6792 (2011).

19

Bunupuradah T, Chetchotisakd P,

Ananworanich $\mathrm{J}$ et al. A randomized comparison of second-line lopinavir/ritonavir monotherapy versus tenofovir/lamivudine/ lopinavir/ritonavir in patients failing NNRTI regimens: the HIV STAR study. Antivir. Ther. 17(7), 1351-1361 (2012).

20 Paton NI, Kityo C, Hoppe A et al.

Assessment of second-line antiretroviral regimens for HIV therapy in Africa. N. Engl. J. Med. 371(3), 234-247 (2014).
21 Rintamaki LS, Davis TC, Skripkauskas S, Bennett CL, Wolf MS. Social stigma concerns and HIV medication adherence. AIDS Patient Care STDs 20(5), 359-368 (2006).

22 Adam's Love.

www.adamslove.org 